\title{
Bioinformatics Challenges and Solutions in Proteomics as Quantitative Methods Mature
}

\author{
Andrew R. Jones
}

$\mathbf{T}$ HIS SPECIAL ISSUE EXAMINES cutting-edge approaches for quantitative proteomics, focusing on bioinformatics challenges and solutions in this complex field. Throughout the history of mass spectrometry (MS)-based proteomic research, the interpretation of data has been challenging. As MS techniques have increasingly become high-throughput, the design and implementation of bespoke software packages and algorithms for statistical analysis have become important areas of research. The field has generally moved towards techniques based on liquid chromatography (LC) directly coupled to a tandem mass spectrometer (LC-MS/MS), used to analyze peptides resulting from digestion of proteins. Approaches can generally be classified as "discovery," in which more proteins are assessed at lower accuracy, or "targeted," often performed by selected reaction monitoring (SRM), in which a smaller number of proteins are more accurately assessed.

In all approaches, the ion intensity signal observed is difficult to predict based on a peptide sequence alone, although analysis of an individual peptide is highly reproducible. As such, a variety of experimental approaches have been designed to calculate the relative abundance of peptides by LC-MS, using differential labels applied prior to sample mixing, or label-free methods on samples run in parallel. These relative signals for peptides can be converted into relative protein abundance values, taking into account ambiguities in the assignment of peptides to proteins, or conflicting evidence from different peptides. Absolute protein abundance can usually only be calculated by incorporating differentially-labeled internal standards (peptides or proteins) in known abundances and converting the unit-less intensities from a relative technique into an absolute value.

This special issue covers a range of important challenges facing the proteome bioinformatics community. It is hugely challenging for software developers to cope with the heterogeneity in proteomics; there are many different experimental methods, different instrument file formats, and different intermediate formats. Many software packages handle only a subset of approaches and they are difficult to connect. A collection of U.K.-based proteome bioinformatics groups have collaborated to construct a detailed review of the state-of-theart in the software provision for quantitative proteomics, and describe a web-based resource that helps researchers to select the appropriate software tools for their local pipeline (Gonzalez-Galarza et al., 2012). The article also briefly de- scribes community data standards under development by the Proteomics Standards Initiative, which will help solve some of the problems of heterogeneity and interconnectivity between software. The issue of software heterogeneity is also tackled in an original study from the group of Lennart Martens, in which the interplay between label-free approaches for quantitation and search engines for peptide/protein identification is analyzed (Degroeve et al., 2012). They demonstrate a significant difference in the results of quantitative analysis through the differential selection of a search engine to use for identification, and they provide recommendations for optimizing these analyses.

Two new software packages are also presented in this issue: Eisenacher and colleagues describe the FindPairs module of the PeakQuant software, targeted towards approaches using ${ }^{15} \mathrm{~N}$ labeling, which is not well covered in the most popular open source tools (Eisenacher et al., 2012); Qi and colleagues describe an open source post-processing tool for using the commercial package Progenesis LC-MS (designed solely for label-free analysis), as a tool for the analysis of stable isotope labeling data and Top3 pseudo absolute quantitation (Qi et al., 2012).

One of the major missing aspects of quantitative proteomics approaches is any detailed understanding of the importance of the efficiency of digestion. It is widely acknowledged that enzymatic digestion of proteins, say with trypsin, is rarely $100 \%$ efficient. For approaches in which proteins are digested into peptides in parallel samples, any variability in peptide digestion will have marked effects on estimates of peptide abundance, and thus these "missed cleavage" peptides are problematic for quantitation. Lawless and Hubbard present a software tool, incorporating support vector machines, capable of accurate prediction of candidate peptide bonds that will be poorly cleaved, leading to missed cleavage peptides (Lawless and Hubbard, 2012).

In targeted approaches based on SRM, particular peptides are selected for analysis based on transitions (triplets of chromatographic retention time, peptide mass/charge, and peptide fragmentation product mass/charge). These transitions are difficult to predict computationally, and so require experimental data to be collected in advance for peptides of interest. Fan and colleagues describe a web-based tool that can help in the design of transitions, called MRMAid 2.0 (Fan et al., 2012). MRMAid 2.0 demonstrates the utility of public

Institute of Integrative Biology, University of Liverpool, Liverpool, U.K. 
data deposition integrated into the new version, in which the authors have included a module for extracting large volumes of data from the PRIDE database at the EBI (http://www .ebi.ac.uk/pride/).

Lastly, the development of advanced statistical approaches is a growing area of importance, since the complexity of proteomic data means standard statistical frameworks are insufficient and bespoke approaches are required. Richardson and colleagues present a bayesian statistical framework for calculating accurate relative peptide and protein abundance values from data that contain a considerable noise component, as is the case for most if not all MS-based approaches (Richardson et al., 2012).

This special issue presents solutions to some of the challenges in quantitative proteomics, but there is still an enormous amount of work to do in this exciting field. Software tools are needed that can evolve in tandem with technical innovation and function robustly even after developers have moved on. Interconnectivity between different pipelines is still challenging, and will remain so until universal file format standards become more widely adopted. Proteomics data are complex and multi-layered, with noise introduced at several different stages at the bench and in silico, for which bespoke statistical tests and normalization methods are currently lacking. Finally, proteomics will benefit from improved benchmarking and comparison of tools, and the release of more gold-standard data sets is undoubtedly needed. This dynamic research field is continuing to grow, and it is likely to present many new and exciting challenges in bioinformatics for many years to come.

\section{Author Disclosure Statement}

The author declares that no conflicting financial interests exist.

\section{References}

Degroeve, S., Staes, A., De Bock, P.-J., and Martens, L. (2012). The effect of peptide identification search algorithms on MS2based label-free protein quantification. OMICS 16, this issue.

Eisenacher, M., Kohl, M., Wiese, S., et al. (2012). Find Pairs-the module for protein quantification of the PeakQuant Software Suite. OMICS 16, this issue.

Fan, J., Mohareb, F., Bond, N., Lilley, K., and Bessant, C. (2012). MRMaid 2.0: Mining PRIDE for evidence-based SRM transitions. OMICS 16, this issue.

Gonzalez-Galarza, F.F., Lawless, C., Hubbard, S.J., et al. (2012). A critical appraisal of techniques, software packages and standards for quantitative proteomic analysis. OMICS 16, this issue.

Lawless, C., and Hubbard, S.J. (2012). Prediction of missed proteolytic cleavages for the selection of surrogate peptides for quantitative proteomics. OMICS 16, this issue.

Qi, D., Brownridge, P., Xia, D., et al. (2012). A software toolkit and interface for performing stable isotope labeling and Top3 quantification using Progenesis LC-MS. OMICS 16, this issue.

Richardson, K., Denny, R., Hughes, C., et al. (2012). A probabilistic framework for peptide and protein quantification from data dependent and data independent LC-MS proteomics experiments. OMICS 16, this issue.

Address correspondence to: Andrew R. Jones Institute of Integrative Biology University of Liverpool The Biosciences Building Crown Street, L69 7ZB Liverpool, U.K.

E-mail: Andrew.Jones@liv.ac.uk 